\title{
A Task Scheduling Algorithm Based on Potential Games in Cloud Computing Environment
}

\author{
Ming-chun Zheng ${ }^{1}$ and Xiao $\mathrm{Li}^{2 *}$ \\ ${ }^{1}$ College of Management Science and Engineering, Shandong Normal University, \\ Jinan, China \\ ${ }^{2}$ State Key Laboratory of Networking and Switching Technology, Beijing \\ University of Posts and Telecommunications, Beijing, China \\ ${ }^{1}$ zhmc163@163.com, ${ }^{2}$ sdnulixiao@126.com
}

\begin{abstract}
Efficient task scheduling mechanism in cloud computing can improve the resource utilization and enhance the overall performance of the cloud computing environment. However, the existing strategies based on static task requirements are difficult to guarantee the stability of the system, while dynamic mechanisms have higher complexity. In this paper, a new task scheduling algorithm based on potential game is proposed. We prove that the potential game will reach Nash equilibrium quickly. Also, the system load balancing level is adaptive with the number of users' task changing. The experimental results show the priority of the proposed algorithm.
\end{abstract}

Keywords: cloud computing, data center, task scheduling, potential games, Nash equilibrium

\section{Introduction}

Cloud computing has emerged as a popular computing model to support on demand services ${ }^{[1]}$, such as Amazon's EC2 ${ }^{[2]}$, Microsoft's Azure ${ }^{[3]}$ and Google's Map-Reduce Programming System ${ }^{[4]}$. Cloud computing provides a variety of resources for users through the service form. Using virtualization technology, it maps physical machines' resources to a virtual machine layer, where virtual machine perform users' tasks. It deploys multiple virtual machines in a physical server. In this way, it not only improves server resources utilization, but also ensures the application independence by different users. With the growing number of servers in data center, resource scheduling problem is becoming a hotspot ${ }^{[5]}$. In cloud computing, one users' application is a task. Task scheduling researches how to map these tasks to appropriate virtual machines. Currently, most task scheduling strategies are solved using intelligent algorithm ${ }^{[6,7,8,9]}$ such as, genetic algorithm, ant colony algorithm and so on. However, these methods have some problems, such as lower ability to search the global optimal solution, poorer convergence and so on. Meanwhile, the degree of system load balancing can't be adaptive to the amount of users' tasks changing.

Considering the weak points of above scheduling algorithms, this paper researches the task scheduling problem in cloud computing environments. The mapping problem of users' task to virtual machine is abstracted into a path selection problem in traffic network. We model a potential game with single population. In this model, each task agent is viewed as a selfish participant. We conduct the disjoint paths from source nodes to destination nodes as participants' strategies. We defined a utility function based on virtual machines' processing delay, and constructed a potential function of this game. We prove that the game would eventually converge to the Nash equilibrium through

*Corresponding author. 
theoretical analysis. Finally, we proposed a task scheduling algorithm based on WolfFrank. All users' tasks will be scheduled by the algorithm. Through our experimental simulation, we conclude that this algorithm can guarantee that the system load balancing level is adaptive to the number of users' task changing. We analyzed users' selection process through simulation. Experimental results show that the game eventually converges to a stable point, which verifies the theoretical analysis. Moreover, we extend our algorithm by adding a constraint, which is in keeping with the realistic scenario.

\section{Related Work}

Some researchers have proposed many intelligent algorithms to solve the task scheduling problem ${ }^{[5]}$. Authors proposed a hybrid scheduling algorithm ${ }^{[6]}$, which combines genetic algorithm and ant colony algorithm. It brought fast global search capabilities of genetic algorithm and simulation resource load capabilities of ant colony algorithm into play, which improved the algorithm solving speed in some extent. However, the degree of system load balancing can't be adaptive to the number of users' task changing.

An improved Particle Swarm Optimization algorithm was proposed ${ }^{[7]}$, in which researchers controlled global and local search by dynamic multi-population collaboration and reverse flight of mutation particle. To some extent, it avoided the algorithm falling into local optimization. But it had a poorer convergence and slower solving.

In recent years, game theory was widely applied to transport network and network routing problems. Some literatures ${ }^{[10,11,12]}$ studied the resource scheduling problem using game theory. Authors solved the resource allocation problem using population game theory ${ }^{[10]}$. Considering each participant is rational and selfish, they proposed an evolutionary algorithm, which took the system optimization and users' fairness into account. Eventually, they proved the resource allocation game always exists Nash equilibrium. A new class of game was defined ${ }^{[11]}$ that is cloud computing resource allocation game (CRAGs). The game could guarantee both optimal prices paid by users and optimal resources usage of cloud data centers. Researchers also proved conditions where the game achieved a variety of stable equilibrium. It was abstracted into an evolution game that the virtual networks choose paths of the underlying physical network ${ }^{[12]}$. The virtual network adjusted their physical path by repeated game, and analyzed the evolution process by potential game theory. Finally, they proved that the evolution results would reach evolutionary equilibrium. Meanwhile, researchers proposed an evolutionary algorithm to achieve the path selection.

\section{Preliminaries}

\subsection{Task Scheduling in Cloud Computing}

In the 21 st century, the scale of cloud data centers is further expanding, and the number of servers is rapidly increasing. In such circumstance, task scheduling is more and more important. Many virtual machines are deployed in physical servers through virtualization technology. Using virtual machines to do users' task can improve the computing power and reliability of data centers.

Resource scheduling in Cloud data centers is divided into two level scheduling. The first level scheduling is task scheduling mentioned in this paper. The goal is how to map multiple tasks to the existing virtual machine. The second scheduling studies how to map virtual machines to physical servers, so that virtual machines can be created or migrated appropriate in physical servers. Figure 1 describes the two level scheduling. 


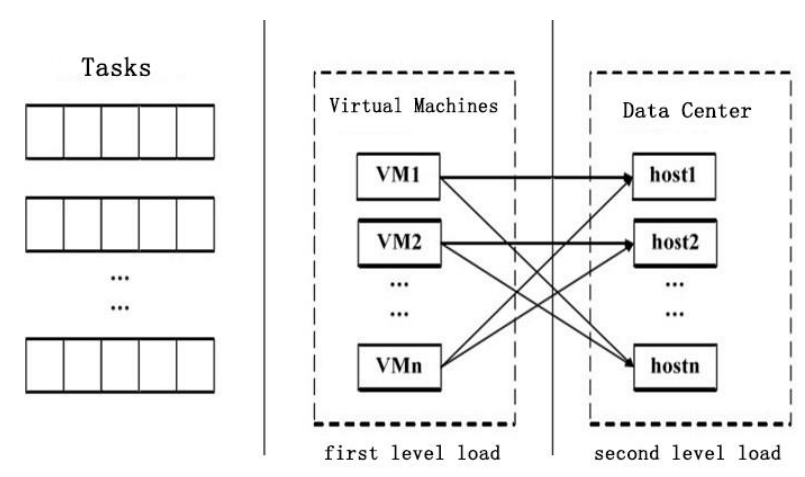

Figure 1. Resources Scheduling Description in Cloud Data Center

\subsection{Potential Games}

The conception of Potential Game with finite players is first proposed by Monderer, Shapeley ${ }^{[13]}$ in 1996, which admits a potential function: a real-valued function defined on the space of pure strategy profiles such that the change in any player's payoffs from a unilateral deviation is exactly matched by the diversification in potential. It follows immediately that Nash equilibria are the local maximizers of potential.

In this paper, we need use a notion of potential games for infinite populations. Potential game with infinite populations is proposed by William H. Sandholm ${ }^{[14]}$. He pointed out that, a game with continuous player sets is a potential game if it admits a potential function: in this context, a real valued function on the space of strategy distributions whose gradient is the vector of payoff functions. That is, we call game $\mathrm{G}$ is a potential game if condition $(\mathrm{P})$ holds:

(P) There exists a function $f(x)$, such that $\frac{\partial f}{\partial x_{i}}=F_{i}(x)$ for all $x, i \in S$. ( $S$ is the space of players strategy, and $i$ is a strategy; $x$ is the strategy distribution.)

Condition (P) says that there is a continuously differentiable function $f$ whose gradient, $\nabla f$, equals the payoff vector $F$. The function $f$, which is unique up to an additive constant, is called the potential function of the game.

In finite player potential games, all equilibria are local maximizers (or minimizers) of potential function. Meanwhile, since better reply adjustment processes increase potential function, all equilibria are locally stable. However, in infinite player settings, these statements are false. Researchers characterize Nash equilibria as the states which satisfy the Kuhn-Tucker first order conditions for a maximizer (or minimizer) of potential function. That is to say, while all maximizers (or minimizers) are equilibria, not all equilibria are maximizers (or minimizers).

\subsection{Introduction of the Cloud Computing}

This paper examines the task scheduling problem in the cloud computing distributed mode. In the cloud data center, there are several local schedulers and a task pool. The submitted application requests are divided into various sub-tasks, then these sub-tasks enter into the task pool. These schedulers are communicating with each other periodically to obtain their load condition. The algorithm's main idea is, all user tasks are a group participant, and there is a game between these participants. They compete with each other for virtual machine resources. Finally, the game will reach a Nash equilibrium, in which no participants can increase their utility by change their own strategy. We can design an algorithm to solve the assignment distribution for each virtual machine. The algorithm results are equal to participants' free competition. The cloud environment is as Figure 2. 


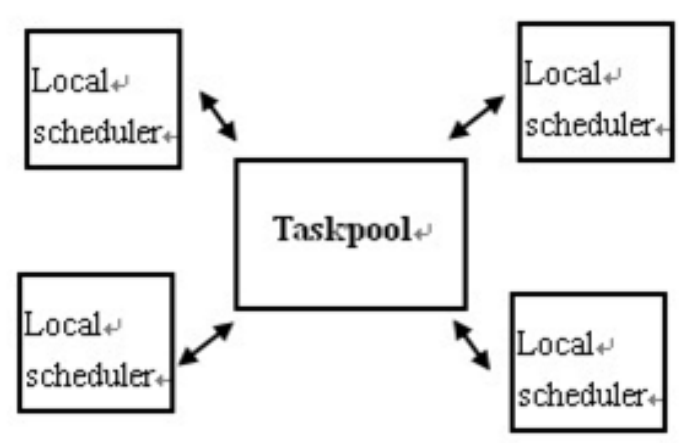

Figure 2. Cloud Environment

\section{The Potential Game Model of Task Scheduling in Cloud Computing Environment}

\subsection{The Task Scheduling Game Model}

A game usually consists of participants, strategy set and utility function. We define our model as $G=<N, S, F>$. We assume this is a single population game.

In this game, we view the users' task agents as participants. Each user task has a task agent, so the task agents set is the participants set, that is, $N=\{1,2, \ldots, i, \ldots n\}$.

In this game, virtual machines are task agents' selectable strategies. Each participant in the population chooses a pure strategy. For a population, its strategy is a mixed strategy, which is the participants' distribution of choosing different virtual machines in the population.

In this single population, the number of task agents is $n(n \geq 1)$, whose strategy set is $S=\{1,2, \ldots m\}$. And $m$ is the amount of strategies. The strategy distribution in this population is $x=\left\{x_{1}, x_{2}, \ldots x_{i} \ldots x_{m}\right\} . x_{i}$ is the number of participants who choose strategy $i$ in this population. It exists the equation, $\sum_{1}^{m} x_{i}=n, m$ is the number of virtual machine.

The utility function of task agents is the processing delay of virtual machines. Task agents always want to reduce their utility, therefore, the strategy which has a lower utility, is more likely to be chosen by users' task agents. We define the virtual machines' processing delay as a function of the amount of tasks above it. The function is as follows:

$$
t_{i}\left(x_{i}\right)=t_{i}^{0} \cdot\left(1+\alpha\left(\frac{x_{i}}{C_{i}}\right)^{\beta}\right)
$$

$x_{i}$ is the amount of tasks in virtual machine $i . C_{i}$ is the maximum amount of parallel processing tasks of virtual machine $i . t_{i}^{0}$ is the basic processing delay of virtual machine $i . \alpha, \beta$ are the coefficient. The utility function of task agent who choose virtual machine ${ }^{i}$ as its strategy is as follows: 


$$
F_{i}(x)=t_{i}\left(x_{i}\right)=t_{i}^{0} \cdot\left(1+\alpha\left(\frac{x_{i}}{C_{i}}\right)^{\beta}\right)
$$

(2)

When all participants can not reduce their utility by changing their strategies, we say that the game reached a Nash equilibrium. According to the concept of Nash, each task agent's choosing strategy is the best response to other participants. In the competing cloud computing environment, each user will get the best results, which is to obtain the virtual machine with the minimum processing delay. Meanwhile, with the increasing of tasks, the degree of system load balancing can be changed.

\subsection{The Task Scheduling Game is a Potential Game}

In the cloud computing environments, we define the system's cost as $f(x)=\sum_{i \in S} \int_{0}^{v_{i}} t_{i}(w) d w$. Now, we will prove that this function is the potential function of the task scheduling game. Meanwhile, the function has the relationship with the equilibrium points of this game.

Theorem 1 The task scheduling game $G$ in cloud computing environments is a potential game, and $f(x)$ is its potential function.

Proof The partial derivative $f(x)=\sum_{i \in S} \int_{0}^{v_{i}} t_{i}(w) d w$ can be obtained:

$$
\begin{aligned}
& \frac{\partial f(x)}{\partial x_{i}}=\frac{\partial \sum_{i \in S} \int_{0}^{x_{i}} t_{i}^{0}\left(1+\alpha \cdot\left(\frac{w}{C_{i}}\right)^{\beta}\right) d w}{\partial x_{i}} \\
& =t_{i}^{0}\left(1+\alpha \cdot\left(\frac{x_{i}}{C_{i}}\right)^{\beta}\right)=F_{i}(x)
\end{aligned}
$$

According to the definition of potential function, we know $f(x)$ is the potential function of the game, and this game is a potential game.

\subsection{The Nash Equilibrium of Task Scheduling Game is Consistent with the Minimum Value of the Potential Game}

In cloud computing environments, the task scheduling game will finally reach to Nash equilibria. We will prove the Nash equilibrium of task scheduling game is consistent with the minimum value of the potential game. We assume a minimum problem as Equation 4.

$$
\min f(x)=\sum_{v=1}^{M} \int_{0}^{x_{i}} t_{i}^{0}\left(1+\alpha \cdot\left(\frac{x_{i}}{C_{i}}\right)^{\beta}\right) d x
$$

$$
\text { s.t. }\left\{\begin{array}{l}
\sum_{i=1}^{M} x_{i}=c \\
x_{i} \geq 0
\end{array}, \forall i \in S_{i}\right.
$$

The Lagrangian for this minimum problem is: 


$$
L(x, \lambda, \mu)=f(x)-\sum_{i=1}^{M} \lambda_{i} \cdot x_{i}-\mu_{i}\left(\sum_{i=1}^{M} x_{i}-c\right)
$$

So the Kuhn-Tucker first-order necessary conditions are:

$$
\begin{aligned}
& \text { (KT1) } \frac{\partial f(x)}{\partial x_{i}}=\lambda_{i}+\mu_{i} \\
& \text { (KT2) } \lambda_{i} \cdot x_{i}=0 \\
& \text { (KT3) } \lambda_{i} \geq 0
\end{aligned}
$$

Theorem 2 The state $x \in X$ is a Nash equilibrium of the potential game $\mathrm{G}$ if and only if $(x, \lambda, \mu)$ satisfies $(K T 1),(K T 2)$ and $(K T 3)$ for some $\lambda, \mu$.

Proof If $x$ is a Nash equilibrium of $\mathrm{G}$, then since $\nabla f(x)=F(x)$, the Kuhn-Tucker conditions are satisfied by $x, \mu_{i}=\min F_{i}(x), \lambda_{i}=F_{i}(x)-\mu_{i} \geq 0, \lambda_{i} \cdot x_{i}=0$.

Conversely, if $(x, \lambda, \mu)$ satisfies the Kuhn-Tucker conditions, then for this population, (KT1) and (KT2) imply that $F_{p}(x)=\nabla f(x)=\mu$ Furthermore, (KT1)and(KT3) imply that $F_{i}(x)=\mu_{i}+\lambda_{i} \geq \mu_{i}$ for any virtual machine $i$. Hence, $F_{i}(x)=\mu_{i}=\min F_{i}(x)$. Thus, when the strategy attribution is $x$, the tasks' utilities are the minimum. So, $x$ is the Nash equilibrium of the game.

\section{Algorithm Design}

From the above analysis, the Nash equilibrium of potential game is consistent with the minimum value of the potential function. Thus, we can solve the Nash equilibrium by the minimum value of the potential function. We design the following algorithm to solve the optimization problem.

Algorithm ABPG is based on the Frank-Wolf for solving. The basic idea is: in each iteration, we first find a steepest descent direction, and then we further find an optimal iteration step size. Finally, we obtain a new starting point of the next iteration based on the steepest descent and the optimal iteration step size. We repeat this iteration by the above method until we find the optimal solution. $\theta^{n}\left(0 \leq \theta^{n} \leq 1\right)$ is obtained by the onedimensional optimization problem, as in Equation 6.

$$
\min f\left(x_{i}^{n}+\theta^{n}\left(y_{i}^{n}\right)\right)=\sum_{i \in S} \int_{0}^{x_{i}^{n}+\theta\left(y_{i}^{n}-x_{i}^{n}\right)} t_{i}^{0}\left(1+\alpha \cdot\left(\frac{w}{C_{i}}\right)^{\beta}\right) d w
$$

$y_{i}^{n}$ is the additional amount of virtual machine task of the nth iteration. $x_{i}^{n}$ is the starting point of the $\mathrm{n}^{\text {th }}$ iteration.

The solution of Equation 6 is

$$
\sum_{i \in S}\left(y_{i}^{n}-x_{i}^{n}\right) \cdot t_{i}^{0}\left[1+\alpha \cdot\left(\frac{x_{i}^{n}+\theta^{n}\left(y_{i}^{n}-x_{i}^{n}\right)}{C_{i}}\right)^{\beta}\right]=0
$$

(7)

And the stopping criterion of this algorithm is, 


$$
\frac{\sqrt{\sum_{i \in S}\left(x_{i}^{n+1}-x_{i+1}^{n}\right)^{2}}}{\sum_{i \in S} x_{i}^{n}} \leq K
$$

(8)

\section{ALGORITHM ABPG}

Step 0 Initialization. We assume the amount of tasks in each virtual machine is zero. Then, we can obtain the utility of each virtual machine. All task agents choose the virtual machine with minimal utility. We obtain the amount of each virtual machine, denoted by $\left\{x_{i}^{1}\right\}$, and make $n=1$.

Step 1 Update the utility of each virtual machine based on $\left\{x_{i}^{1}\right\}$. Make $t_{i}^{n}=t_{i}^{0}\left[1+\alpha\left(\frac{x_{i}^{n}}{C_{i}}\right)^{\beta}\right] \forall i \in S$.

Step 2 Determine the direction of the next iteration. All task agents choose the virtual machine with minimal utility, obtained by $\left\{t_{i}^{n}\right\}$. Obtain an additional amount of virtual machine task, denoted by $\left\{y_{i}^{n}\right\}$.

Step 3 Determine the iteration step size. Find $\theta_{n}$ satisfied Eq. 7.

Step 4 Update the amount of virtual machine tasks by $x_{i}^{n+1}=x_{i}^{n}+\theta^{n}\left(y_{i}^{n}-x_{i}^{n}\right) \forall i \in S$.

Step 5 Convergence test. If $\left\{x_{i}^{n+1}\right\}$ satisfies Equation 8, the algorithm stop. And $\left\{x_{i}^{n+1}\right\}$ is the Nash equilibrium solution. Else, make $n=n+1$, return to Step 1 .

The degree of system load balancing can be adaptive to the amount of users' task changing by algorithm ABPG. That is to say, when receiving 10000 tasks, the cloud data center uses 50 virtual machines in a load balancing state; when receiving 30000 tasks, the cloud data center turns to use 100 virtual machines which are in a load balancing. It is the greatest advantage relative to other task scheduling algorithms.

\section{Experimental Analysis}

In order to verify the reliability and validity of the proposed algorithm, we make experimental simulation using cloud computing simulation software--CloudSim. We extends the Datacenter-Broker in order to implement our algorithm. We use C\# to realize our algorithm. In our simulation, we set 30 virtual machines in the cloud data center.

\subsection{The Comparison of Total Tasks Run-time}

We simulate the total task run-time and the number of tasks of each Virtual Machine. We assume the utility function of each virtual machine is:

$$
t_{i}^{n}=a\left[1+\alpha\left(\frac{x_{i}^{n}}{b}\right)^{\beta}\right]
$$

The parameters are set $\alpha=0.15, \beta=10$. 
Table 1. Parameters List

\begin{tabular}{ccccccccccc}
\hline $\boldsymbol{V M}$ & $\mathbf{1}$ & $\mathbf{2}$ & $\mathbf{3}$ & $\mathbf{4}$ & $\mathbf{5}$ & $\mathbf{6}$ & $\mathbf{7}$ & $\boldsymbol{8}$ & $\mathbf{9}$ & $\mathbf{1 0}$ \\
\hline $\boldsymbol{a}$ & 0.1 & 0.6 & 0.2 & 0.2 & 0.25 & 0.4 & 0.58 & 0.35 & 0.42 & 0.38 \\
\hline $\boldsymbol{b}$ & 300 & 200 & 250 & 200 & 300 & 500 & 600 & 400 & 100 & 200 \\
\hline $\boldsymbol{V M}$ & $\mathbf{1 1}$ & $\mathbf{1 2}$ & $\mathbf{1 3}$ & $\mathbf{1 4}$ & $\mathbf{1 5}$ & $\mathbf{1 6}$ & $\mathbf{1 7}$ & $\mathbf{1 8}$ & $\mathbf{1 9}$ & $\mathbf{2 0}$ \\
\hline $\boldsymbol{a}$ & 0.5 & 0.25 & 0.3 & 0.4 & 0.15 & 0.4 & 0.5 & 0.2 & 0.1 & 0.25 \\
\hline $\boldsymbol{b}$ & 500 & 400 & 150 & 600 & 800 & 250 & 500 & 400 & 150 & 180 \\
\hline $\boldsymbol{V M}$ & $\mathbf{2 1}$ & $\mathbf{2 2}$ & $\mathbf{2 3}$ & $\mathbf{2 4}$ & $\mathbf{2 5}$ & $\mathbf{2 6}$ & $\mathbf{2 7}$ & $\mathbf{2 8}$ & $\mathbf{2 9}$ & $\mathbf{3 0}$ \\
\hline $\boldsymbol{a}$ & 0.5 & 0.25 & 0.4 & 0.4 & 0.15 & 0.4 & 0.2 & 0.3 & 0.4 & 0.15 \\
\hline $\boldsymbol{b}$ & 600 & 200 & 400 & 200 & 350 & 600 & 100 & 140 & 430 & 330 \\
\hline
\end{tabular}

We analysis the CloudSim3.0 task scheduling algorithm, max-min task scheduling algorithm, min-min algorithm and the proposed algorithm in this paper. The comparison figure of the four algorithms of different number of tasks run-time is in Figure 3.
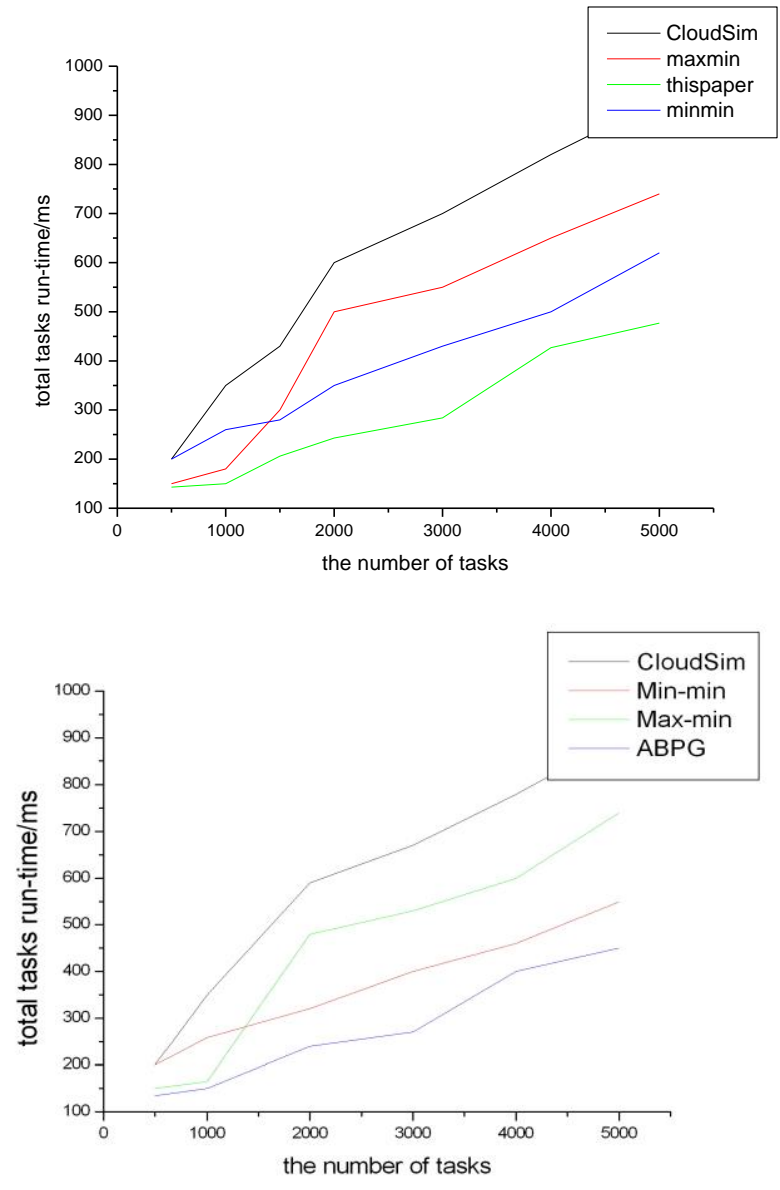

Figure 3. Tasks Run-timeComparison 
From Figure 3, we can see that, no matter how many tasks, the task run-time of our algorithm (ABPG) is shorter than CloudSim algorithm, max-min and min-min. Furthermore, the more the tasks, the greater advantage of our algorithm.

\subsection{Tasks Load Distribution}

When the number of tasks is 2000, 3000, 4000 and 5000, each virtual machine's tasks distribution is in Figure 4, Figure 5, Figure 6 and Figure 7.

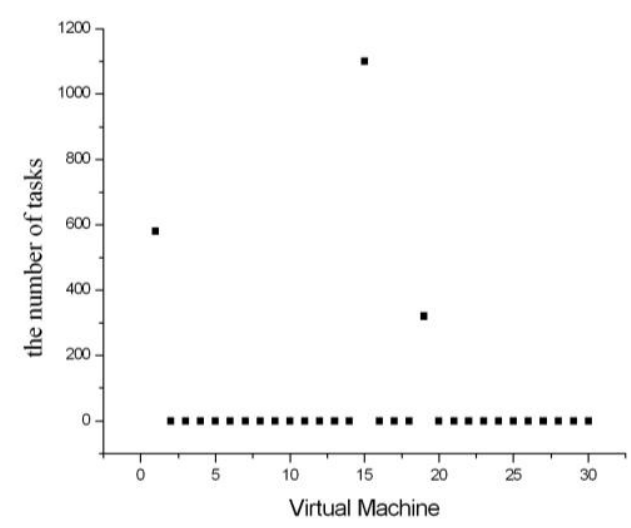

Figure 4. Each Virtual Machine's Tasks Distribution (2000)

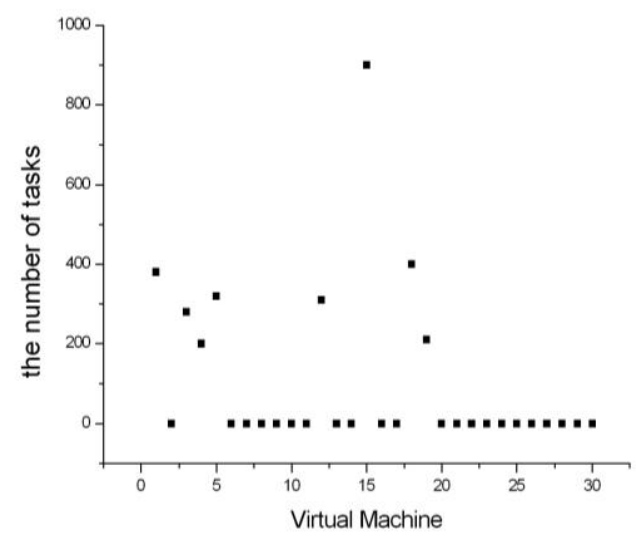

Figure 5. Each Virtual Machine's Tasks Distribution (3000)

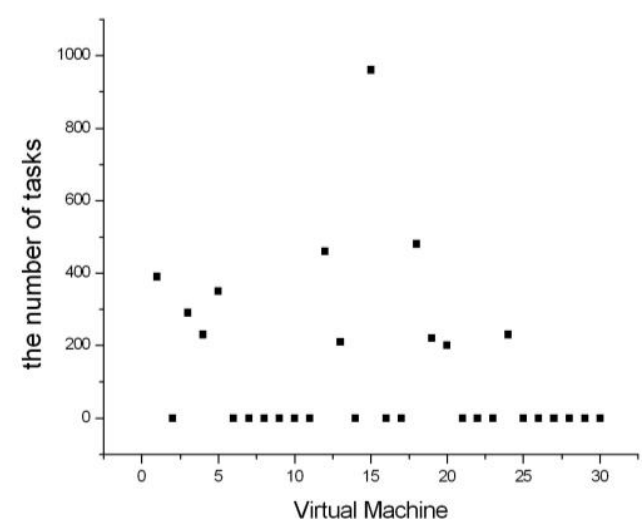

Figure 6. Each Virtual Machine's Tasks Distribution (4000) 
From Figure 4 to Figure 7, we can see each virtual machines' load state. When we have 2000 tasks in the cloud data center, the designed algorithm assigns only 3 virtual machines to do these tasks; When we have 3000 tasks in the cloud data center, it assigns 8 virtual machines to do these tasks; When we have 4000 tasks, it assigns 11 virtual machines to do these tasks; When we have 5000 tasks, it assigns 13 virtual machines to do these tasks. With the growth of the number of tasks, more and more tasks are assigned to virtual machines. The degree of system load balancing can be adaptive to the number of users' task changing based on the designed algorithm. Then, from the experiment, we conclude that the run-time of each virtual machine which has tasks is equal to the minimum time of all virtual machines. Finally, the potential game reaches to Nash equilibrium.

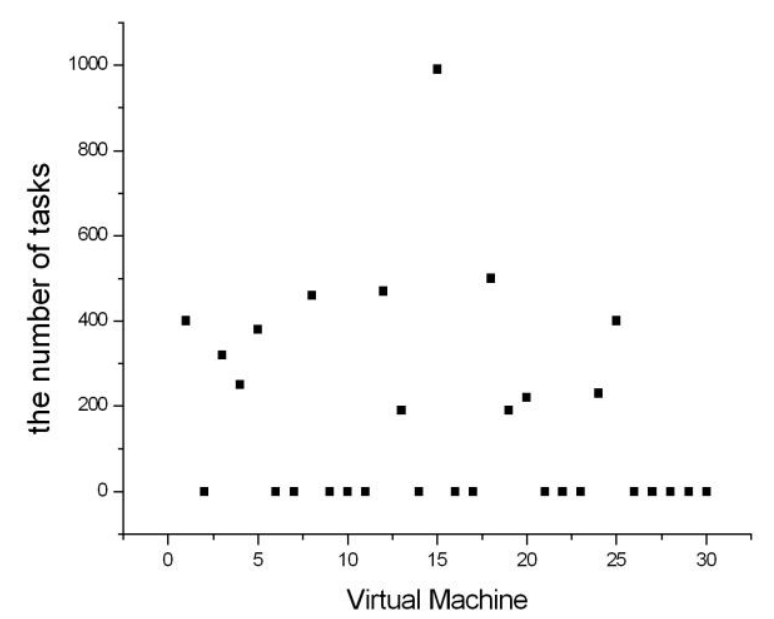

Figure7. Each Virtual Machine's Tasks Distribution (5000)

\section{Algorithm Extended}

The section 4 shows that, the task scheduling game is a potential game. And the potential function is $f(x)=\sum_{i \in S} \int_{0}^{x_{i}} t_{i}(w) d w$. Also, we prove that the minimum of the potential function is equivalent to the Nash equilibrium.

In the cloud data centers, each virtual machine $i$ has a certain threshold limit, which regulates the amount of tasks on the virtual machine. In our model, we are talking about the heterogeneous situation of virtual machines. That is, threshold value of each virtual machine may be different. Some virtual machines can handle more tasks while others less.

Minimum problem of potential function is equivalent to the following the optimization problem, which is the Equation 4.

In the optimization problem (4), we do not consider the threshold of the virtual machines. In the utility function, $C_{i}$ is the threshold value of virtual machine $i$. That is to say, the assignment tasks of virtual machine $i$ should not surpass the $C_{i}$. However, in our designed algorithm, we do not consider the constraint. For more general, we add the constraint to optimization problem (4), then it turns to optimization problem (9).

$$
\min f(x)=\sum_{i} \int_{0}^{x_{i}} t_{i}^{0}\left(1+\alpha \cdot\left(\frac{x_{i}}{C_{i}}\right)^{\beta}\right) d x
$$




$$
\text { S.t. }\left\{\begin{array}{l}
\sum_{i \in S} x_{i}=c \\
x_{i} \geq 0, \forall i \in S \\
x_{i} \leq C_{i}, \forall i \in S
\end{array}\right.
$$

In the task allocation model with threshold limit of the virtual machine, we can't simply use the designed algorithm to solve the optimization problem. In fact, we assume the constraint $x_{i} \leq C_{i}$ has an operator $d_{i}$. Then the delay of virtual machine $i$ turns to $\tilde{t}_{i}\left(x_{i}\right)=t_{i}\left(x_{i}\right)+d_{i}$. That is, add $d_{i}$ to the delay of virtual machine $i$. This time, we can think $d_{i}$ is a queuing delay. The actual scenario is, each task agents can select a virtual machine which has no congestion, but the processing delay of these machines is longer; on the other aspect, they can select a congestion virtual machine. In these machines, the processing delay is shorter, however, because of the more tasks on these machines, tasks need to queue in the task queue. In this way, users can achieve the optimal principle, which is the Wardrop equilibrium theory. The delay of all selected virtual machines is equal and equals to the minimum delay. In Figure 8, we can see the queuing exists only if the amount of tasks surpass the threshold value of the virtual machine.

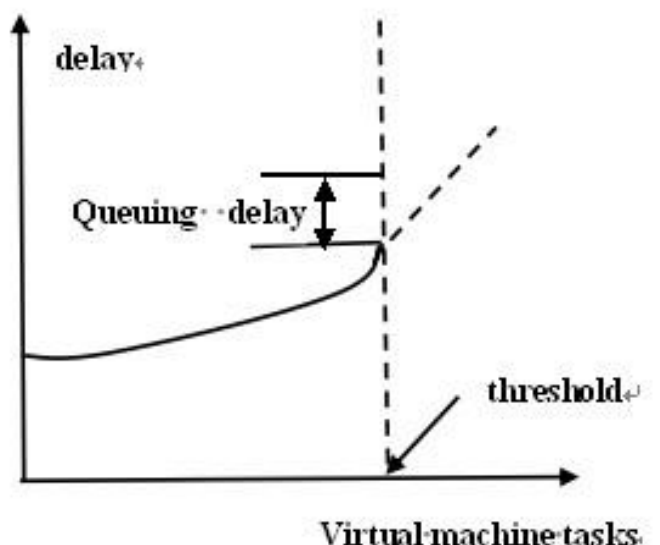

Figure 8. Queuing Delay when Virtual Machine Reach to Threshold Value

Consequently, we extended our designed algorithm as EABPG.

\section{Algorithm EABPG}

Step 0 Initialization. Select the initialization value of ${ }^{\mu}$ for each virtual machine.

$$
\mu_{i}^{0}=\left\{\begin{array}{lc}
\left\{t_{i}\left(x_{i}^{0}\right)-t_{i}\left(C_{i}\right),\right. & x_{i}^{0}>C_{i} \\
0, & \text { else }
\end{array}\right.
$$

$x_{i}^{0}$ is the solution of algorithm ABPG. Set $\rho^{1}(>0)$, and iteration index $n=1$.

Step 1 Solve the Sub-problem. Set the utility of each virtual machine $i$ to $t_{i}\left(x_{i}\right)+\max \left\{0, \mu_{i}^{n}+\rho^{n}\left(x_{i}-C_{i}\right)\right\}$, and use algorithm ABPG to solve. 
Step 2 If $\max _{i \in S}\left\{\frac{\left|x_{i}^{n}-C_{i}\right|}{C_{i}} \frac{\left|x_{i}^{n}-C_{i}\right|}{C_{i}} \mid \mu_{i}^{n}>0\right\}<\varepsilon \quad$ ( $\varepsilon$ is a small default number $)$, we stop iteration. $\left(x^{n}, \mu^{n}\right)$ is the solution of the optimization problem (9). Else, return to Step 3.

Step 3 Update Multiplier and Penalty Parameter. Update multiplier $\mu_{i}^{n+1}$ by Equation (10); Update penalty parameter $\rho^{n+1}$ by Equation (11).

$$
\mu_{i}^{n+1}=\max \left\{0, \mu_{i}^{n}+\rho^{n}\left[x_{i}\left(\rho^{n}, \mu^{n}\right)-C_{i}\right]\right\}, i \in S
$$

$$
\rho^{n+1}=\left\{\begin{array}{l}
\kappa \rho^{n}, \sqrt{\sum_{i \in S} \max ^{2}\left\{-\mu_{i}^{n} / \rho^{n}, x_{i}^{n}-C_{i}\right\}} \\
>\gamma \sqrt{\sum_{i \in S} \max ^{2}\left\{-\mu_{i}^{n-1} / \rho^{n-1}, x_{i}^{n-1}-C_{i}\right\}} \\
\rho^{n}, \quad \text { else }
\end{array}\right.
$$

In equation (11), the values of parameters are $2 \leq \kappa \leq 10, \lambda=0.25$.

Make $n=n+1$, and return to Step 1 .

\section{Conclusion}

In the paper, the task scheduling problem of Cloud computing is modeled as a potential game. Each task agent is a selfish participant. Task agents compete with each other for virtual machine resources. We prove that this game has a Nash equilibrium at least. Meanwhile, the Nash equilibrium of task scheduling game is consistent with the minimum value of the potential game. Then we design an algorithm to solve the optimization problem. The experimental results show the priority of the algorithm. Then, we extend our algorithm by adding a constraint, which is in keeping with the realistic scenario. The main innovation of this paper is: Our algorithm ABPG (EABPG) has a shorter run-time than the existing algorithms. Moreover, the degree of system load balancing can be adaptive to the amount of users' task change.

\section{Acknowledgements}

This work was supported by National Natural Science Foundation of China (61402266), Shandong Province Natural Science Foundation (ZR2012FM013) .

\section{References}

[1] "Group of virtualization and cloud computing", Virtualization and Cloud Computing,

Publishing House of Electronics Industry, Beijing, (2009).

[2] Introduction of Amazon Web Services [EB/OL], http//aws.amazon.com.

[3] Microsoft, Azure Service Platform Overview, J. INSIGHT (Microsoft), vol. 2, (2008), pp. 1-23.

[4] D. Jeffrey and C. Sanjay, "MapReduce: simplified data processing on large clusters", OSDI'04:

6th Symposium on Operating Systems Design and Implementation, (2004), pp. 137-149. 
[5] Q. F. Qian, C. L. Li, X. Q. Zhang and L. Y. Li, "Virtual resources review of Cloud data center", Application Research of Computers, vol. 29, no. 7, (2012), pp. 2411-2415.

[6] Y. Zhang, F. Li and T. Zhou, "The task scheduling research in Cloud Computing based on genetic ant colony algorithm", Computer Engineering and Applications (2012).

[7] W. J. Liu, M. H. Zhang and W. Y. Guo, "The Cloud Computing resource scheduling strategy based on MPSO algorithm", Computer Applications, vol. 37, no. 11, (2011), pp. 43-44, 48.

[8] J. P. Luo, X. Li and M. R. Chen, "The resource scheduling based on Shuffled Frog Leaping Algorithm”, Computer Engineering and Applications, vol. 48, no. 29, (2012), pp. 67-72.

[9] Y. Liu and Z. W, "Zhao, The resource scheduling strategy based on Optimal Genetic Algorithm in Cloud Computing environments", Journals of Beijing Normal University, vol. 48, no. 4, (2012), pp. 373-384.

[10] G. Y. Wei, "A game-theoretic method of fair resource allocation for cloud computing services", Supercomput, vol. 54, (2010), pp. 252-269.

[11] J. Virajith and N. Giang, et al, "Cloud Resource Allocation Games", University of llinois, http://hdl.handle.net/2142/17427,(2010)

[12] Y. Yang, S. Z. Chen and X. Li, "The research of dynamic selection of the underlying resource based on Evolutionary Games in Network Virtualization environments", Journals of Communication, vol. 33(Z2), (2012), pp. 25-349.

[13] D. Monderer and L. Shapley, "Potential Games, Games and Economic Behavior 14", (1996), pp. 124-143.

[14] H.S. William, "Potential Games with Continuous Player Sets", Journal of Economic Theory, vol. 97, no. 1, (2001), pp. 81-108.

[15] T. Heikkinen, "A potential game approach to distributed power control and scheduling", Computer Networks, vol. 50, (2006), pp. 2295-2311.

[16] H. Yang and H. -J. Huang, "The multi-class, multi-criteria traffic network equilibrium and systems optimum problem”, Transportation Research Part B, (2004), pp. 1-15.

[17] G. Karakostas and A. Viglas, "Equilibria for networks with malicious users", Mathematical Programming, vol. 110, no. 3, (2007), pp. 591-613.

[18] E. Koutscoupias and C. H. Papadimitriou, "Worst-case equilibria", Proceedings of the 16th Annual Symposium on Theoretical Aspects of Computer Science (STACS), (1999), pp. 404413.

[19] E. Altman, H. Hameda, "Evolutionary dynamics and potential games in non-cooperative routing", The 5th Intermational Symposium on Modeling and Optimization in Mobile, Ad Hoc and Wirless Networks and Workshops (2007), Limassol, Cyprus.

[20] Shakkottais, Altman and Kumara, "The case for non-cooperative multihoming of users to access points in IEEE 802.11 WLANs", IEEE I n focom (2006), Barcelona, Spain,

[21] The Cloud Computing and Distributed Systems (CLOUDS) Laboratory, University of Melbourne. CloudSim [EB/OL], 2012-01-11,http://www.cloudbus.org/cloudsim/

[22] T. Roughgarden, "Designing Networks for Selfish Users is Hard", Journal of Computer and System Sciences, vol. 72, no. 5, (2006), pp. 922-953.

[23] W. Wang, G. S. Zeng and D. Z. Tang, "Cloud-DLS: Dynamic trusted scheduling for Cloud computing", Expert Systems with Applications, vol. 39, no. 3, (2012), pp. 2321-2329.

[24] R. Braun and H. Siegel, "A comparison of eleven static heuristics for mapping a class of independent tasks onto heterogeneous distributed computing systems", J of Parallel and Distributed Computing, vol. 61, no. 6, (2001), pp. 810-837.

[25] S. Rizos and H. Zhao, "A Hybrid Heuristic for DAG Scheduling on Heterogeneous Systems", In: Proceedings of Parallel and Distributed Processing Symposium UK, (2004), pp. 26-30.

\section{Authors}

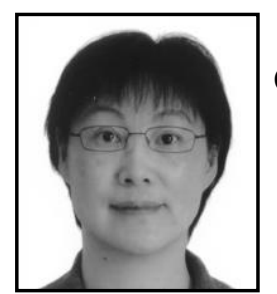

Ming-Chun Zheng. Born in 1963, Professor, zhmc163@163.com, Computer Network, Overlay Network, Network Virtualization. 
International Journal of Future Generation Communication and Networking

Vol. 8, No. 1 (2015)

Xiao Li. Born in 1988, Master, sdnulixiao@126.com, Cloud

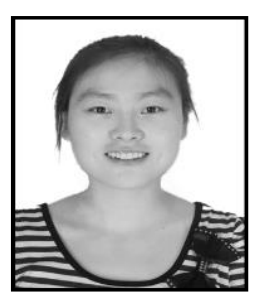
Computing, Network Virtualization 